origin", but psychological improvement following hysterectomy would be either unexpected or, if it occurred, coincidental.

The possibility exists that for a significant number of these women their "menorrhagia" might have been an expression of psychological vulnerability rather than uterine pathology and this is supported by the absence of uterine pathology from so many of them, the infrequency of anaemia and the fact that a considerable number of them remained psychologically disturbed following hysterectomy. This explanation also accords with the findings of a study in preparation, in which 62 per cent of women who complained of menorrhagia had little evidence of significant menstrual bleeding, but considerable evidence of psycho-social disturbance.

The danger with a hysterectomy being performed for the complaint of menorrhagia is not that women with significant bleeding will become depressed as a result, but that this operation may be inflicted upon depressed women with little evidence of abnormal bleeding.

St Bartholomew's Hospital,

West Smithfield, London ECIA $7 B E$

\section{MaUrice Greenberg}

\section{ANXIETY MANAGEMENT TRAINING}

\section{DeAR SiR,}

I should like to make three brief comments on the paper by Ramm et al on "anxiety management training . . ." (Journal, April 1982, 140, 367-73). Firstly, no matter what was recorded in the patients' diaries, do the authors really believe that the negative self-instruction group religiously repeated such self-depreciatory and pessimistic comments ? Secondly, I was under the impression that in clinical trials a putative treatment is compared with the best available or, at worst, a placebo rather than a noxious procedure.

Finally, I consider it unethical to ask patients to repeatedly make such statements as "really going crazy ... going to make a fool of myself . . . getting steadily worse ..." when in stressful situations, and am most surprised that the MRC supported the project.

Gartnavel Royal Hospital,

$$
\text { J. A. G. WATT }
$$

1055 Great Western Road, Glasgow G120XH

Dr Watt's letter seems to assume that patients are very unwilling to repeat self-depreciatory or pessimistic comments, but we have not found this to be so in our own experience. During exposure treatment not a few patients even spontaneously try out such methods without being asked to do so. Some report the approach to be helpful, and there are many anecdotes of the therapeutic effect of such paradoxical intention, especially in the writings of Victor Frankl. His classic advice to patients who had anxiety about their heart was 'go out and have a heart attack right now', and I myself have found this approach calming during realistic danger. There was thus good reason to believe that negative self-instruction, far from being a 'noxious procedure', might well reduce anxiety occasionally. Our results bore this out to some extent and found no untoward effects from the approach. However, there is still no 'best available' treatment for anxiety states which is demonstrably better than placebo.

Current treatments of phobias and compulsive rituals by exposure in vivo are effective but were also initially thought by many to be too unethical to try. Only when clinicians carefully explored what actually happened rather than prejudge the issue was this significant advance made in treatment. Such experiments are an essence of clinical research, provided that the effects are always carefully monitored, with the patient's wellbeing constantly in mind.

Institute of Psychiatry,

ISAAC MARKS

De Crespigny Park, Denmark Hill, London SE5 8 AF

\section{GENETIC LINKAGE ANALYSIS AND AGE-OF-ONSET CORRECTION}

DeAR SiR,

In his otherwise excellent article on 'The Search for Genetic Linkage in Schizophrenia' (Journal, May 1982, 140, 432-37), Dr Watt makes some rather misleading statements concerning a linkage analysis of Huntington's disease (HD) published by Brackenridge et al (1978), a paper of which I was a co-author. We did not report linkage between $H D$ and haptoglobin as suggested by Dr Watt, but rather a maximum lod of 1.88 (at $\theta=0.05$ ). This is suggestive of linkage but in the lod score method, linkage is conventionally not "proved" until a maximum lod in excess of 3.0 is obtained.

Quoting Hodge et al (1980), Dr Watt claims our investigation did not employ a sufficient correction to account for young individuals in our pedigrees who were not showing HD but could be presymptomatic carriers of the abnormal gene concerned. Our investigation was in fact the first published HD linkage analysis to employ such a correction and it was done on the basis of each subject's age and the population distribution of onset age; the same method was employed by Hodge et al. Dr Watt suggests the 